\title{
New solvent-stabilized few-layer black phosphorus for antibacterial
}

\section{applications}

Zhenyu Sun, ${ }^{1, *}$ Yuqin Zhang, ${ }^{1 \dagger}$ Hao Yu, ${ }^{2 \dagger}$ Chao Yan, ${ }^{3}$ Yongchao Liu, ${ }^{3}$ Song Hong, ${ }^{2}$

Hengcong Tao, ${ }^{1}$ Alex W. Robertson, ${ }^{4}$ Zhuo Wang ${ }^{2, *}$ Agílio A. H. Pádua ${ }^{5}$

${ }^{1}$ State Key Laboratory of Organic-Inorganic Composites, Beijing University of Chemical Technology, Beijing 100029, China

${ }^{2}$ Beijing Advanced Innovation Center for Soft Matter Science and Engineering, State Key Laboratory of Chemical Resource Engineering, College of Science, Beijing University of Chemical Technology, Beijing 100029, China

${ }^{3}$ School of Material Science \& Engineering, Jiangsu University of Science and Technology, Zhenjiang 212003, China

${ }^{4}$ Department of Materials, University of Oxford, Oxford OX1 3PH, United Kingdom

${ }^{5}$ Institute of Chemistry of Clermont-Ferrand, Université Clermont Auvergne \& CNRS, 63000

Clermont-Ferrand, France

${ }^{\dagger}$ These authors contribute equally to this work.

E-mail: sunzy@mail.buct.edu.cn, and wangzhuo77@mail.buct.edu.cn

Keywords: black phosphorus; exfoliation; solvent; antibacterial property

\begin{abstract}
Discovering highly efficient, environmentally friendly, and low-cost exfoliating media that can both disperse and protect black phosphorus (BP) remains a challenge. Herein, we demonstrate such a new molecule, $N, N^{\prime}$-dimethylpropyleneurea (DMPU), for effective exfoliation and dispersion of two-dimensional BP nanosheets. A very high exfoliation efficiency of up to $16 \%$ was achieved in DMPU, significantly surpassing other good solvents. Exfoliated flakes are free from structural disorder or oxidation. Nanosheets retain high stability in DMPU even after addition of 25 vol.\% of common solvents. The solvation shell appears to protect the nanosheets from reacting with water and air, more remarkably than the best solvent $N$-cyclohexyl-2-pyrrolidone reported so far. Molecular dynamics simulations of the exfoliation process show that DMPU is among the effective solvents, although energetically it does not appear as favorable as some other amides. We also demonstrate that our exfoliated BP nanosheets exhibit excellent antimicrobial activities against both Escherichia coli and Staphylococcus aureus, outperforming other common two-dimensional materials of graphene and $\mathrm{MoS}_{2}$,
\end{abstract}


suggesting promise in biomedical applications.

\section{Introduction}

As a new homoatomic two-dimensional (2D) semiconductor since the discovery of graphene, single layered black phosphorus (BP), termed phosphorene, is currently the focus of research interest. ${ }^{[1-4]}$ Unlike graphene with zero band gap, ${ }^{[5]}$ and transition metal dichalcogenides such as $\mathrm{MoS}_{2}, \mathrm{MoSe}_{2}, \mathrm{MoTe}_{2}, \mathrm{WS}_{2}$, and $\mathrm{WSe}_{2}$ that display indirect (dipole forbidden, symmetry forbidden) band gap in thick layers, ${ }^{[6]} \mathrm{BP}$ exhibits an intrinsic direct (dipole allowed, symmetry allowed) band gap in mono- and multilayered forms. Thinning BP to a few atomic layers induces a significant bandgap widening from $\sim 0.3 \mathrm{eV}$ in the bulk to $\sim 2.1 \mathrm{eV}$ for a monolayer, due to quantum size effects. $^{[7]}$ The exciton binding energies, optical absorption spectrum, and linear polarization energy window of BP can be also broadly tuned by tailoring the number of stacked layers. BP exhibits an orthorhombic, buckled crystal structure with two bonds along the orthogonal in-plane directions, providing anisotropy in the mechanical, thermal, electrical, and optical properties. Notably, phosphorene possesses a highly anisotropic hole mobility of $\sim 1000 \mathrm{~cm}^{2} \mathrm{~V}^{-1} \mathrm{~s}^{-1}$ and a large on/off current ratio of $\sim 10^{4}-10^{5} \cdot{ }^{[8-9]}$ Such unique properties render this $2 \mathrm{D}$ material especially intriguing for electronic and optoelectronic devices, ${ }^{[10-11]}$ sensing, ${ }^{[12-15]}$ catalysis, ${ }^{[16-21]}$ solar cells, ${ }^{[22]}$ rechargeable batteries, ${ }^{[23-25]}$ and photothermal therapeutic agents, ${ }^{[26-29]}$ etc.

Production of high-quality BP nanosheets in large quantities is essential for successful applications. ${ }^{[30]}$ However, in contrast to simple van der Waals solids such as graphene ( $\sim 61 \mathrm{meV}$ per $\mathrm{C}$ atom), BP is characterized by stronger interlayer cohesion $(\sim 151 \mathrm{meV}$ per $\mathrm{P}$ atom), resulting from significant charge redistribution between layers facilitated by the electron lone pairs. ${ }^{[31-32]}$ The lone pairs also contribute to high chemical reactivity. These aspects render it more complex and challenging to delaminate layers from bulk BP. Liquid-phase exfoliation ${ }^{[33-37]}$ to produce $2 \mathrm{D}$ BP appears to be a facile and potentially scalable route. Exfoliated nanosheets can be noncovalently stabilized against re-aggregation by interaction with a variety of solvents, ${ }^{[23,32,38-46]}$ surfactants, ${ }^{[47]}$ polymers, ${ }^{[48]}$ or ionic liquids ${ }^{[49-50]}$ (see Table S1). 
Despite their success, we note that surfactants, polymers and ionic liquids are thirdphase dispersants, and must be removed after processing for a range of applications. In addition, it would be much simpler to work only with two-phase dispersions, i.e. solvent and nanosheet. However, dispersion and exfoliation of BP nanosheets have been only achieved in a very limited number of solvents. The yield of BP nanosheets afforded by liquid exfoliation and centrifugation (CF) is currently rather low, being mostly less than $10 \%$. This drives ongoing efforts to exploit and develop new systems to accomplish higher exfoliation efficiency. Another major challenge is that phosphorene dispersions are chemically unstable in many solvents, such as water, isopropanol (IPA), and $\gamma$ butyrolactone $(\mathrm{GBL}){ }^{[45]}$ thus hampering prospective applications. Hanlon et al. demonstrated successful exfoliation of BP in $N$-cyclohexyl-2-pyrrolidinone (CHP), yielding stable phosphorene nanosheets in ambient conditions. ${ }^{[42]}$ Warren and coworkers explored 18 solvents for BP exfoliation and concluded that benzonitrile (BZ) was the best one, giving the highest concentration $\left(0.11 \mathrm{mg} \mathrm{mL}^{-1}\right)$ after $\mathrm{CF}^{[43]}$ However, the level of oxidation or of other sonolysis surface modification effects of exfoliated nanosheets in BZ and other solvents after exposure to air are still unclear. The best dispersion solvents (N-methylpyrrolidone (NMP), BZ) for BP reported hitherto are toxic and relatively costly. ${ }^{[43]}$ The development of highly efficient, environmentally friendly, and practical exfoliating media is, therefore, critically required for dispersion processing of BP. It is also significant to find a solvent that inhibits phosphorene from oxidative decomposition to a great extent, providing efficient protection.

Molecular dynamics (MD) simulations provide a molecular-scale view into the exfoliation process, allowing us to resolve different effects at play. It is key to understand the role of interactions, i.e. the competition between the solvent-solvent and inter-sheet forces on one side and the solvent-material interactions on the other, the ordering of solvent molecules in the interfacial layers, the role of molecular shape that can favor intercalation between sheets, and also confinement effects. ${ }^{[32]}$ An atomistic model of BP and phosphorene was developed recently ${ }^{[32]}$ in which the van der Waals and orbital overlap contributions to inter-layer cohesion (discussed above) are carefully parameterized. Among the quantities calculated through simulation, the free energy cost 
(reversible work) associated with peeling a single phosphorene layer from a stack of BP allowed comparison of different solvents, leading to agreement to experiments ${ }^{[38,40,42]}$ on exfoliation efficacy. It was found that solvents with planar molecules, such as NMP, could intercalate easily, so molecular shape is an important descriptor for a good solvent, in addition to properties such as polarity, cohesive energy or surface tension.

Here we demonstrate a new and harmless solvent for $\mathrm{BP}, N, N^{\prime}-$ dimethylpropyleneurea (DMPU). BP can be efficiently exfoliated and stabilized in DMPU via bath ultrasonication. We show that a significantly higher level of exfoliation is achieved in this solvent than in any other. We also investigate the exfoliation process of BP in DMPU using molecular dynamics simulations, using the force field for BP derived from $a b$ initio calculations lattice dynamics. ${ }^{[32]}$ Exfoliated BP nanosheets exhibit a low oxidation level of only 4.3 atom $\%$ even after 7 days of ambient exposure. The dispersions remain stable with only $<5 \%$ degradation after 3 days even when 25 vol.\% fraction of hexane or acetone is added. The solvation shells of DMPU provide effective passivation against degradation with better efficiency than the well-known solvent CHP in the presence of air and water. We use our dispersions further to explore antibacterial properties of DMPU-stabilized phosphorene by cell viability assays together with scanning electron microscopy (SEM). On the basis of these results, we introduce BP nanosheets as a new family of 2D antimicrobial nanomaterials.

\section{Results and discussion}

Exfoliation and characterization. Benchmarking the values of dispersion concentration remains one of the biggest issues in the BP community because the final concentration and flake size vary substantially from one group to another. Such a scenario is due to the fact that different experimental conditions, such as initial bulk concentration, ultrasonication power or applied shear force, temperature, etc., are not specified in some cases. To compare like with like, we made BP dispersions in varying solvents under identical conditions. All BP dispersions prior to exfoliation were prepared in a glovebox to avoid effects of air. After being subjected to mild bath 
ultrasonication $(400 \mathrm{~W}, 40 \mathrm{kHz}$ ) followed by $\mathrm{CF}$, stable phosphorene dispersions in DMPU and the 7 most successful solvents, including NMP, CHP, benzonitrile (BZ), 1,3-dimethyl-2-imidazolidinone (DMI), N,N-dimethyl formamide (DMF), dimethyl sulfoxide (DMSO), and IPA, were readily obtained (Fig. 1a). As a control, distilled water was also checked for BP dispersion but nearly all flakes sedimented after CF. Propylene carbonate (PC) and bio-based levulinic acid (LA), which haven't been reported for phosphorene exfoliation, were found to exfoliate and disperse BP at reasonable concentrations. Undoubtedly, this expands the library of available exfoliating solvents with new members, which should be very useful for further processing and applications of BP. Note that the polar aprotic solvent DMPU is substantially more effective in exfoliating BP than the other tested solvents (Fig. 1b). In stark contrast to $<8 \%$ of exfoliation efficiency in these best known good solvents, the yield of BP nanosheets is approximately 16\% (initial BP concentration, $C_{\mathrm{I}}: 1 \mathrm{mg}$ $\mathrm{mL}^{-1}$; bath ultrasonication: $3 \mathrm{~h}$; $\mathrm{CF}: 3000 \mathrm{rpm}, 30 \mathrm{~min}$ ). The Tyndall effect of the dispersion in DMPU confirmed its colloidal nature (Fig. S1a). Thermogravimetric (TGA) analysis (Fig. S1b) together with knowledge of the mass of (BP + DMPU) after evaporation of DMPU for known volumes of dispersions allowed determination of stock dispersion concentration. A sample of the stock dispersion was serially diluted with DMPU. A linear trend was observed for the optical absorbance per unit-cell length at $465 \mathrm{~nm}\left(A_{465} / l\right)$ versus concentration $\left(C_{\mathrm{BP}}\right)$, whereby the absorption coefficient at 465 $\mathrm{nm}$ was derived to be $a_{465} \approx 31.5 \mathrm{~cm}^{2} \mathrm{mg}^{-1}$ based on the Lambert-Beer law $(A=\alpha C l)$ (Fig. S1c). This value is larger than the one $\left(\sim 15.1 \mathrm{~cm}^{2} \mathrm{mg}^{-1}\right)$ reported for BP dispersion in CHP by Coleman and co-workers. ${ }^{[42]}$ We used this measured absorption coefficient of $\sim 31.5 \mathrm{~cm}^{2} \mathrm{mg}^{-1}$ to estimate the concentration of all subsequent dispersions.

$C_{\mathrm{BP}}$ was measured versus $C_{\mathrm{I}}$. We found that the dispersion concentration $\left(C_{\mathrm{BP}}\right)$ increased steadily with $C_{\mathrm{I}}$, reaching up to $\sim 1.11 \mathrm{mg} \mathrm{mL}^{-1}$ at $C_{\mathrm{I}}=10 \mathrm{mg} \mathrm{mL}^{-1}$ (Fig. S1d). To the best of our knowledge, this is the highest BP dispersion concentration reported to date.

Optical absorbance spectra and optical absorbances at $465 \mathrm{~nm}, A_{465}(t)$ before and after CF, are shown in Figure S2. The effective optical absorption spectra before CF, 
given in Fig. S2a of the Supporting Information, approach an apparent maximum absorbance level at $465 \mathrm{~nm}$ of absorbance units per $\mathrm{cm}$ (path length) ( $\sim 20$ per $\mathrm{cm}$, Fig. S2c), although further slow increase in absorbance may occur with much longer ultrasonication, as has been observed in graphene and BP dispersed in PVP/ethanol solution. ${ }^{[48]}$ If all of the BP flakes in this dispersion were exfoliated into thin layers, we would expect to see an absorbance leveling off at $\sim 31.5$ per $\mathrm{cm}$, consistent with the absorption coefficient derived in Fig. S1c for a suspension of $1 \mathrm{mg}$ of BP/mL at 465 $\mathrm{nm}$. This discrepancy is explained mostly by the presence of thicker and readily sedimenting BP flakes in our "before-centrifugation" dispersion of Fig. S2a. When we centrifuge to get the spectra and absorption data of Fig. S2b and d, $\sim 73 \%$ of the BP flakes sedimented, including large particulates and an unknown fraction of thick multilayers. The absorption spectra we see in the supernatant is characteristic of small and thin flakes, as studied and reported by Hanlon, Backes, and co-workers. ${ }^{[42]}$ We note that our extinction coefficient of $\sim 20 \mathrm{~cm}^{2} \mathrm{mg}^{-1}$ is close to the $26.5 \mathrm{~cm}^{2} \mathrm{mg}^{-1}$ reported by Hanlon and co-workers after CF. Our estimate of $\sim 31.5 \mathrm{~cm}^{2} \mathrm{mg}^{-1}$ obtained after CF (Fig. $\mathrm{S} 1 \mathrm{c})$, is about twice of the $15.1 \mathrm{~cm}^{2} \mathrm{mg}^{-1}$ reported earlier. Such difference is still unknown. More experimental work, as done for graphene by Nair and co-workers, ${ }^{[51]}$ and theoretical modeling are needed. Fig. S3 illustrates increase in absorbance as a function of $t^{1 / 2}$. Two discernible domains of behavior were seen: a long-time behavior $(t>12 \mathrm{~h})$ and an initial time behavior. These two domains display slopes of $0.0028 \mathrm{~s}^{-1 / 2}$ $(t<12 \mathrm{~h})$ and $0.00095 \mathrm{~s}^{-1 / 2}(t>12 \mathrm{~h})$. Such a scenario suggests that these kinetic processes are diffusion-controlled, and the exfoliation appears rate-limiting as diffusion-controlled processes. The slopes of the two similar domains observed for graphene in water, $0.0026 \mathrm{~s}^{-1 / 2}$ and $0.00086 \mathrm{~s}^{-1 / 2}$, respectively, are about $7.7 \%$ and $10.5 \%$ lower, indicating possibly slower exfoliation of graphene. ${ }^{[52]}$ 
(a)

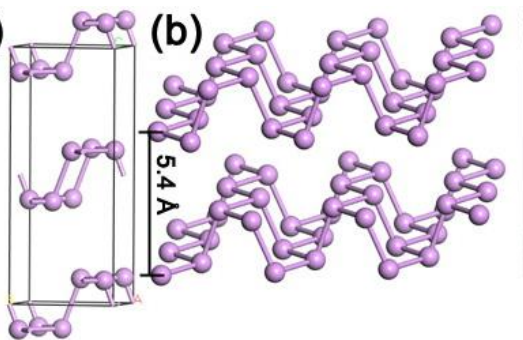

(c)

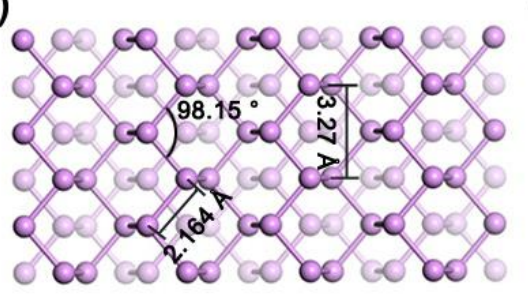

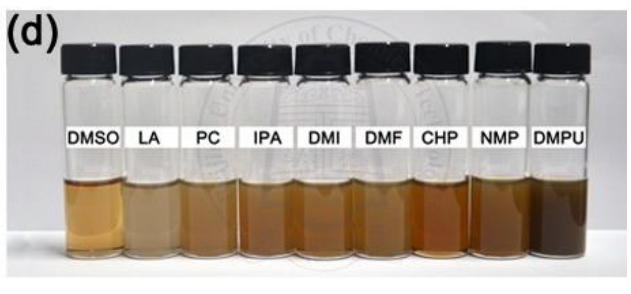

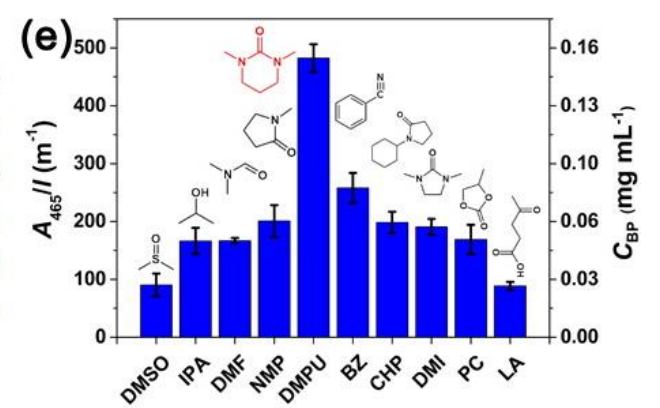

Figure 1. (a) The orthorhombic unit cell of $\mathrm{BP}(a=3.31 \AA, b=4.38 \AA, c=10.50 \AA, \alpha$ $=\beta=\gamma=90^{\circ}$; space group Bmab; crystallography open database ID: 1010325). (b) Two-layer phosphorene, highlighting the interlayer distance of $5.4 \AA$. (c) Top view of BP. (d) Photographs, and (e) $A_{465} / l$ and $C_{\mathrm{BP}}$ for phosphorene dispersions in DMPU and other good solvents (initial BP concentration $C_{\mathrm{I}}=1 \mathrm{mg} \mathrm{mL}^{-1} ; t_{\text {sonic }}=3 \mathrm{~h} ; \mathrm{CF}: 3000 \mathrm{rpm}$, $30 \mathrm{~min})$.

Scanning electron microscopy (SEM) (Fig. 2a, b, and Fig. S4a, d, e) along with energy-dispersive X-ray analysis (Fig. S4b, c, and f) and transmission electron microscopy (TEM) (Fig. S5a) and high-angle annular dark field scanning TEM (HAADF-STEM) (Figs. S5b and 2e) confirmed the successful exfoliation of BP in DMPU, yielding a large number of thin flakes with lateral dimensions of $100 \mathrm{~nm}-4$ $\mu \mathrm{m}$. On the basis of SEM examination, the mean width and length for 311 individual exfoliated flakes were estimated to be about 1200 and 2200 nm, respectively (Fig. 2c and d). We note that the lateral sizes of these nanosheets are bigger than those typically observed for other 2D materials $(<1 \mu \mathrm{m})$. By TEM, thick multi-layer sheets (more than 10 layers) were rarely observed. Fig. 2e shows a HAADF-STEM image of several possibly stacked nanosheets. The corresponding EDX mapping (Fig. $2 \mathrm{f}$ and $\mathrm{g}$ ) shows that the nanosheets are composed of uniform $\mathrm{P}$ element. High-resolution TEM (HRTEM) imaging of different regions revealed that the resulting nanosheets retain 
high quality, free from visible impurities or structural disorder (defects or vacancies). Fig. 3a shows an aberration-corrected HRTEM image of a nanosheet with intact lattice. Lattice fringes of 0.315 , and $0.435 \mathrm{~nm}$ well match those of BP crystals. The fast Fourier transform (FFT), taken from the area enclosed in the square in Fig. 3a, displays the (200), (002), (110), (100) and (001) plane families of orthorhombic BP (Fig. 3b). This indicates that the BP nanosheets exfoliated by bath ultrasonication in DMPU preserve their crystalline nature. The intensity ratio of the (110) and (200) peaks $\left(I_{110} / I_{200}\right)$ is shown to be greater than one, suggesting the structure to be likely a monolayer. ${ }^{[44,53]}$
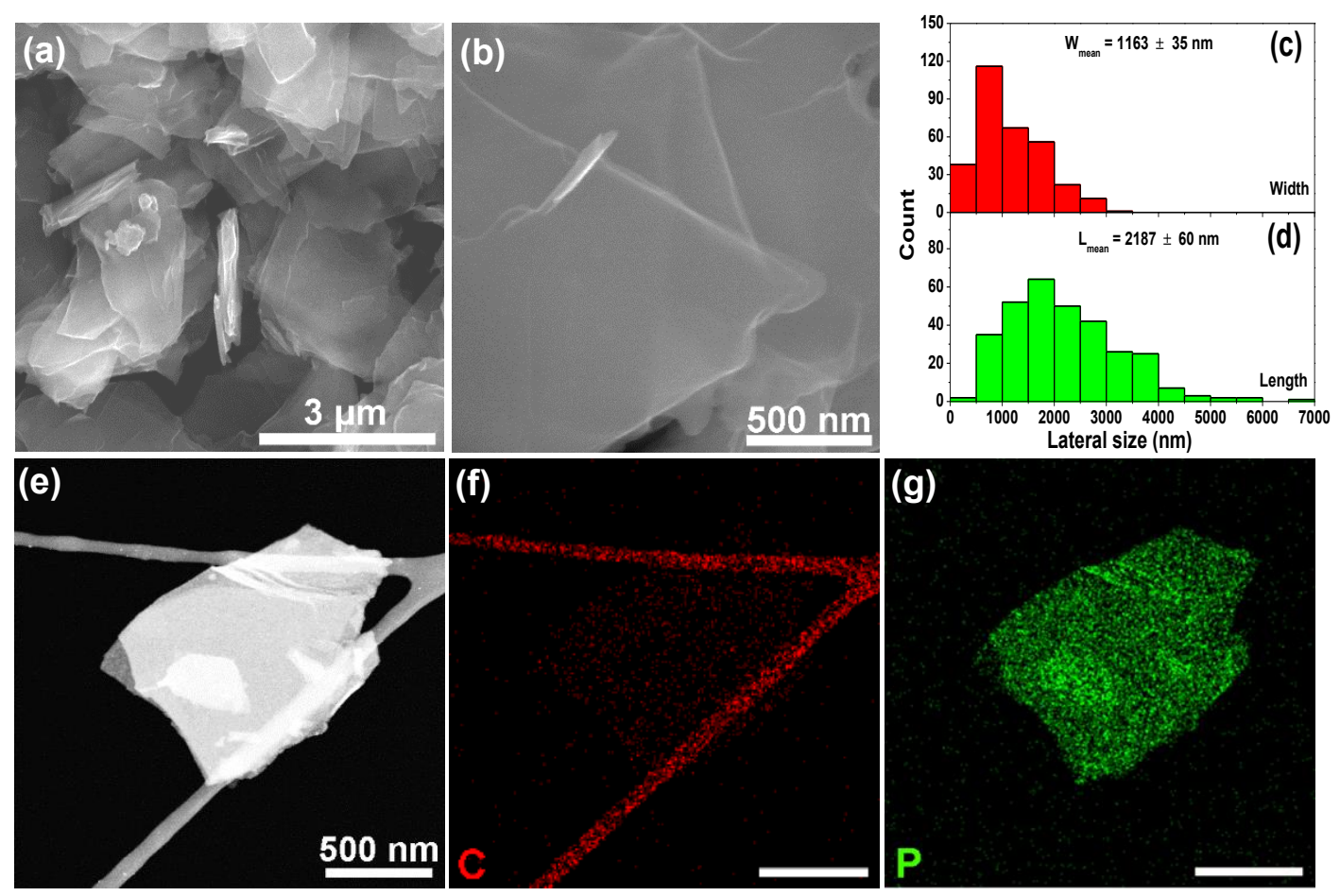

Figure 2. (a) Low- and (b) high-magnification SEM images of exfoliated BP nanosheets $\left(C_{\mathrm{I}}=1 \mathrm{mg} \mathrm{mL}^{-1} ; t_{\text {sonic }}=3 \mathrm{~h} ; \mathrm{CF}: 3000 \mathrm{rpm}, 30 \mathrm{~min}\right)$. (c) Width, and (d) length histograms for 311 randomly chosen flakes obtained by SEM observation. (e) HAADF-STEM image of two possibly stacked BP nanosheets. EDX elemental maps of (f) carbon and $(\mathrm{g})$ phosphorus taken from the region shown in e.

Atomic force microscopy (AFM) analysis showed that the thickness values range from 2.0 to $15.4 \mathrm{~nm}$ (Fig. 3c-i and Fig. S6). The lateral dimensions of the BP flakes agree well with the SEM images. No bubbles, droplets, or other signs of BP degradation 
were observed. Apparent AFM heights from liquid-exfoliated samples are usually overestimated because of residual solvent and effects of capillary forces and adhesion. ${ }^{[42]}$ Approximately $13.9 \%$ of measured flakes have apparent AFM height of about $2.2 \mathrm{~nm}$ that are likely monolayers (Fig. 3c, f, and i). That the AFM-measured thickness value of a single-layer $2 \mathrm{D}$ crystal on a $\mathrm{SiO}_{2} / \mathrm{Si}$ wafer is higher than the theoretical value has been reported in graphene, $\mathrm{MoS}_{2}$, and $\mathrm{BP}{ }^{[54]} \mathrm{Bi}$-layer and fewlayer nanosheets were also observed, as shown in Fig. 3e and Fig. 3d, respectively. Notably, $85.0 \%$ of the flakes have fewer than five layers.
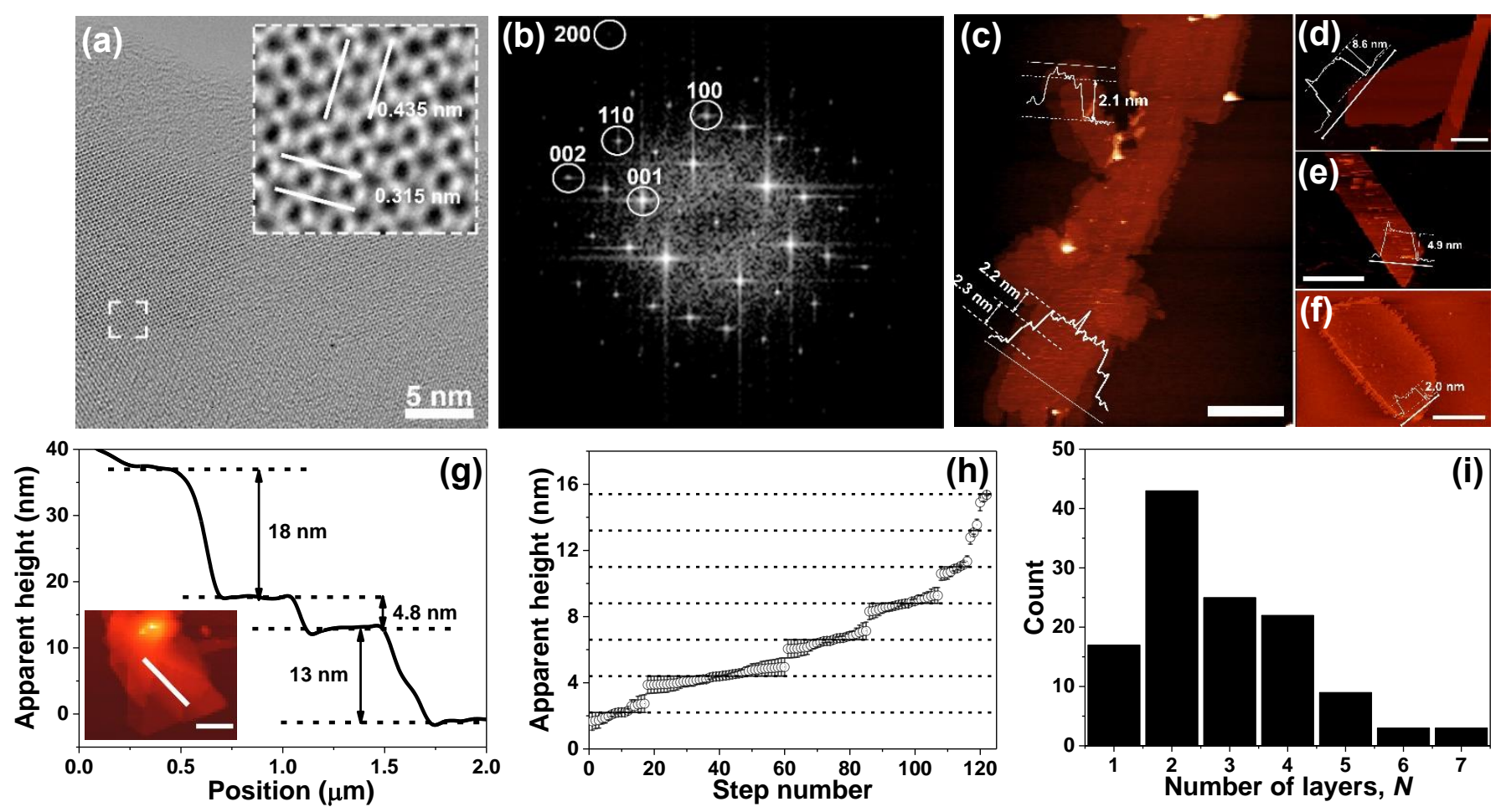

Figure 3. (a) Aberration-corrected HRTEM image of a single sheet. Inset: Enlarged image taken from the selected region in (a). (b) FFT of image shown in (a). (c)-(f) AFM images of single- and few-layer BP flakes with corresponding height profiles. The scale bar in image $\mathrm{c}$ is $2 \mu \mathrm{m}$, and the scale bars in images d-f are $1 \mu \mathrm{m}$. (g) Height profile of an origami-like BP flake in the inset along the line showing clearly resolvable terraces and orientations due to its folding (scale bar, $1 \mu \mathrm{m}$ ). (h) Heights of 120 steps of deposited BP nanosheets in an ascending order. The step height was found to be a multiple of $2.2 \mathrm{~nm}$, which was inferred to be the apparent height of one monolayer. (i) 
Histogram of number of monolayers per nanosheet derived by AFM on 122 flakes.

Raman spectra of bulk BP and exfoliated flakes both show three active modes at frequencies $\sim 362.5,439.1$, and $466.6 \mathrm{~cm}^{-1}$ that can be assigned to an out-of-plane vibrational symmetry $\mathrm{Ag}_{\mathrm{g}}{ }^{1}$, and two in-plane symmetries of $\mathrm{B}_{2 \mathrm{~g}}$ and $\mathrm{Ag}_{\mathrm{g}}{ }^{2}$, respectively (Fig. 4a). ${ }^{[8,42,55]}$ A slight shift of both $\mathrm{Ag}_{\mathrm{g}}{ }^{1}$ and $\mathrm{Ag}_{\mathrm{g}}{ }^{2}$ peaks to higher frequency numbers $\left(\sim 1.4 \mathrm{~cm}^{-1}\right.$ for $\mathrm{A}_{\mathrm{g}}{ }^{1} ; \sim 1.8 \mathrm{~cm}^{-1}$ for $\left.\mathrm{Ag}_{\mathrm{g}}{ }^{2}\right)$ occurred as has been observed for few-layer BP, originating from less hindered oscillation of $\mathrm{P}$ atoms as a result of reduced interlayer van der Waals forces. ${ }^{[56]}$ A spatial Raman map of $\mathrm{Ag}_{\mathrm{g}}{ }^{1} / \mathrm{Ag}^{2}$ integrated peak ratio is presented in Fig. 4b. It has been proposed that the intensity ratio of the $\mathrm{Ag}_{\mathrm{g}}{ }^{1} / \mathrm{Ag}_{\mathrm{g}}{ }^{2}$ phonon is sensitively associated with sample oxidative degradation. As such, we analyzed 209 individual Raman spectra after baseline subtraction. The resultant intensity ratio histogram, as plotted in Fig. 4c, illustrates predominant values of $\mathrm{Ag}_{\mathrm{g}}{ }^{1} \mathrm{Ag}^{2}>0.2$, highlighting low oxidation levels. ${ }^{[55]}$
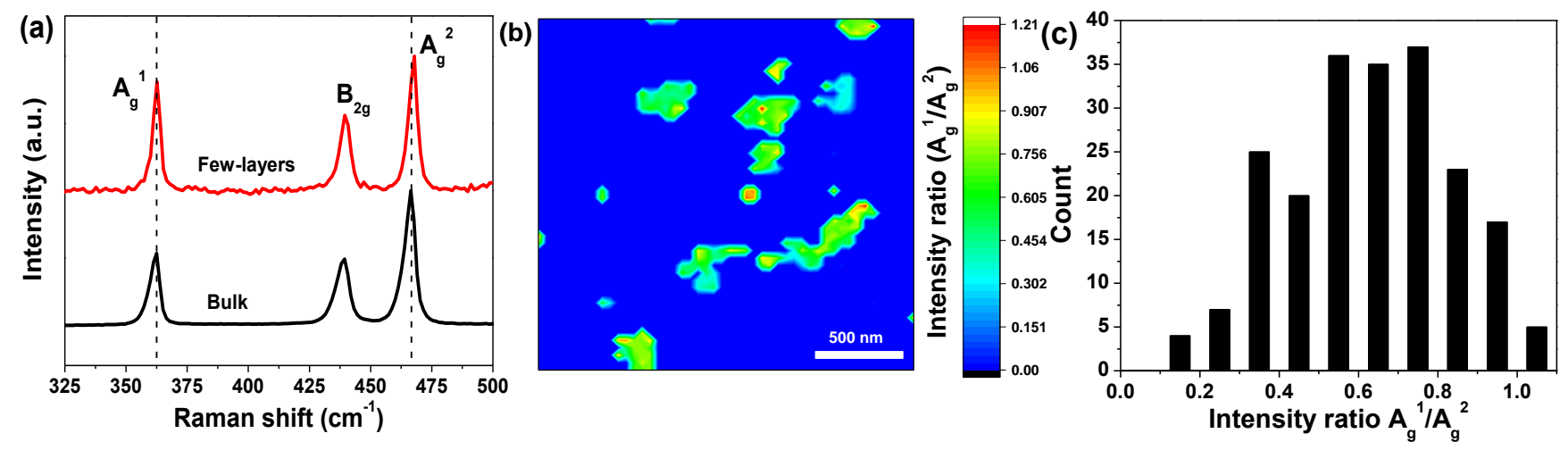

Figure 4. (a) Raman spectra of bulk BP and exfoliated BP nanosheets. (b) Raman $\mathrm{Ag}_{\mathrm{g}}{ }^{1} / \mathrm{Ag}_{\mathrm{g}}{ }^{2}$ integrated peak ratio map of phosphorene dispersions deposited on a $300 \mathrm{~nm}$ thick $\mathrm{SiO}_{2} / \mathrm{Si}$ wafer. (c) Histogram of $\mathrm{Ag}_{\mathrm{g}}{ }^{1} / \mathrm{Ag}^{2}$ integrated peak ratios.

Molecular dynamics simulations. The simulation setup and conditions are identical to those reported by Sresht et al., ${ }^{[32]}$ namely the simulation conditions and the force field used to describe intra-sheet and inter-sheet terms, leading to the mechanical properties of the material and to the cohesion between sheets. Briefly, the periodic 
simulation box contained a stack of four sheets in BP structure, with lateral dimensions of $5.1 \times 4.0 \mathrm{~nm}$, immersed in 1150 DMPU molecules. After equilibration at $300 \mathrm{~K}$ the simulation box has dimensions of $8.0 \times 6.5 \times 5.5 \mathrm{~nm}$, meaning that a thickness of at least $2.5 \mathrm{~nm}$ of solvent is present between stacks, for a cutoff of the short range interactions of $1.2 \mathrm{~nm}$, sufficient to avoid artifacts due to the periodic boundary conditions. The OPLS-AA force field ${ }^{[57]}$ was used to model the structure and interactions of DMPU with parameters from amides and pyrrolidones. The interactions between DMPU and P atoms were described by geometric combining rules considering only the van der Waals part of the BP force field. MD trajectories were obtained using the LAMMPS software. ${ }^{[58]}$ The potential of mean force (PMF) corresponding to pulling the top sheet of the stack was calculated by umbrella sampling, at a series of equilibrium separations, and the weighted histogram analysis method was used to compute the PMF curve. Details can be found in the publication by Sresht et al. ${ }^{[32]}$ The results of the peeling "computer experiment" in DMPU are plotted in Fig. 5.

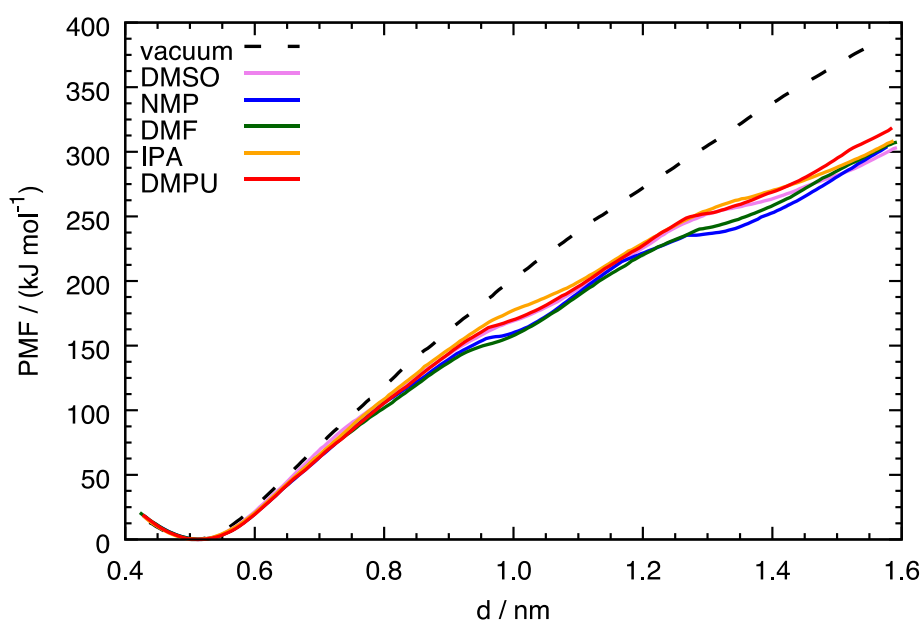

Figure 5. Potential of mean force (PMF) profiles corresponding to the reversible work of peeling the top phosphorene sheet from a stack in the presence of solvent, compared to the same process in vacuum. The waves at ca. 0.9 and $1.3 \mathrm{~nm}$ correspond to the more difficult separation of interlayer P-P contacts at each corrugation (pucker) of phosphorene. DMPU (calculations of this work) is compared to other solvents. ${ }^{[32]}$

The PMF profiles are similar in the different solvents, although NMP and 
dimethylformamide (DMF) show lower PMF values than DMPU, so simulation predicts that although DMPU is among the good solvents for BP exfoliation it is not the best. Because the force field for DMPU is from the same source as for the other solvents, sharing many parameters with DMF and NMP, namely Lennard-Jones potentials and partial charges, it is unlikely that the discrepancy with experiment comes from the interaction potentials

Dispersion stability. To facilitate practical applications, dispersions with high concentration and good stability are preferred. We thus assessed the stability of our phosphorene dispersions under inert conditions by monitoring $A_{465} / l$ versus sedimentation time. Fig. 6a shows the sedimentation profile for a phosphorene dispersion with $C_{\mathrm{BP}} \approx 0.12 \mathrm{mg} \mathrm{mL}^{-1}$. Although some degree of re-aggregation occurred, $\sim 91 \%$ of BP nanosheets in the dispersion still remained stably dispersed over long periods up to $336 \mathrm{~h}$. The curve can be fitted to a monoexponential decay, $C=C_{0}+\left(C_{\mathrm{T}^{-}}\right.$ $\left.C_{0}\right) \mathrm{e}^{-t / \tau}$, where $C_{0}$ represents the concentration of stably dispersed BP, $C_{\mathrm{T}}$ represents the initial concentration of BP, and $\tau$ represents the sedimentation time constant. Fitting the data gave $C_{0}=0.1 \mathrm{mg} / \mathrm{mL}$, and $\tau=43.77 \mathrm{~h}$. This suggests that phosphorene dispersions of $0.1 \mathrm{mg} / \mathrm{mL}$ can retain stability against sedimentation over a reasonable long time. Moreover, no sedimentation of the dispersion was observed, even after exposure to low $\left(-5^{\circ} \mathrm{C}\right)$ or high $\left(80^{\circ} \mathrm{C}\right)$ temperatures for $12 \mathrm{~h}$. The absorbance of the dispersion as well as the spectral shape remained unchanged regardless of freezing treatment (Fig. S7). There is only a very slight decrease in absorbance after heating at $80^{\circ} \mathrm{C}$ for $12 \mathrm{~h}$.

It is known that $\mathrm{BP}$ oxidizes and converts to $\mathrm{P}_{\mathrm{x}} \mathrm{O}_{\mathrm{y}}$ species which can further react with moisture to form phosphoric acid. ${ }^{[55,59-60]}$ It has been envisaged that those ambient adsorbates (e.g., $\mathrm{O}$ and $\mathrm{H}_{2} \mathrm{O}$ ) first interact with $\mathrm{BP}$ noncovalently, and then react covalently to perturb molecular orbitals and degrade its electronic properties. ${ }^{[61]}$ To probe whether chemical modifications take place in exfoliated BP samples upon ambient exposure, we performed X-ray photoelectron spectroscopy (XPS) analysis. The P $2 p$ XPS core-level spectra manifest a single spin-orbit P $2 p^{3 / 2}$ and P $2 p^{1 / 2}$ doublet (spin-orbit splitting of $\sim 0.9 \mathrm{eV}$ ) at $\sim 129.5$ and $130.4 \mathrm{eV}$, respectively, characteristic of 
crystalline $\mathrm{BP}$ (Fig. 6b). Very small oxidized phosphorus (i.e., $\mathrm{P}_{2} \mathrm{O}_{4}$ or $\mathrm{P}_{2} \mathrm{O}_{5}$ moieties) sub-bands are seen at $\sim 134 \mathrm{eV}$, likely stemming from oxygen defects or surface suboxides in the BP that are introduced during exfoliation. ${ }^{[62]}$ It is worth noting that despite 7 days of ambient exposure, the BP nanosheets have a low oxide content of $c a$. 4.3 atom $\%$, close to PVP-stabilized BP. This contrasts to a $\mathrm{P}_{\mathrm{x}} \mathrm{O}_{\mathrm{y}}$ content of $\sim 15 \%$ reported for BP nanosheets in CHP. ${ }^{[42]}$ The oxidation level is also remarkably less compared with those BP nanosheets in IPA and GBL. ${ }^{\left[{ }^{45]}\right.}$ Additionally, no increase in the full-width at half maximum (FWHM: $c a .1 .5 \mathrm{eV}$ ) was observed, suggesting that the long range order was maintained.
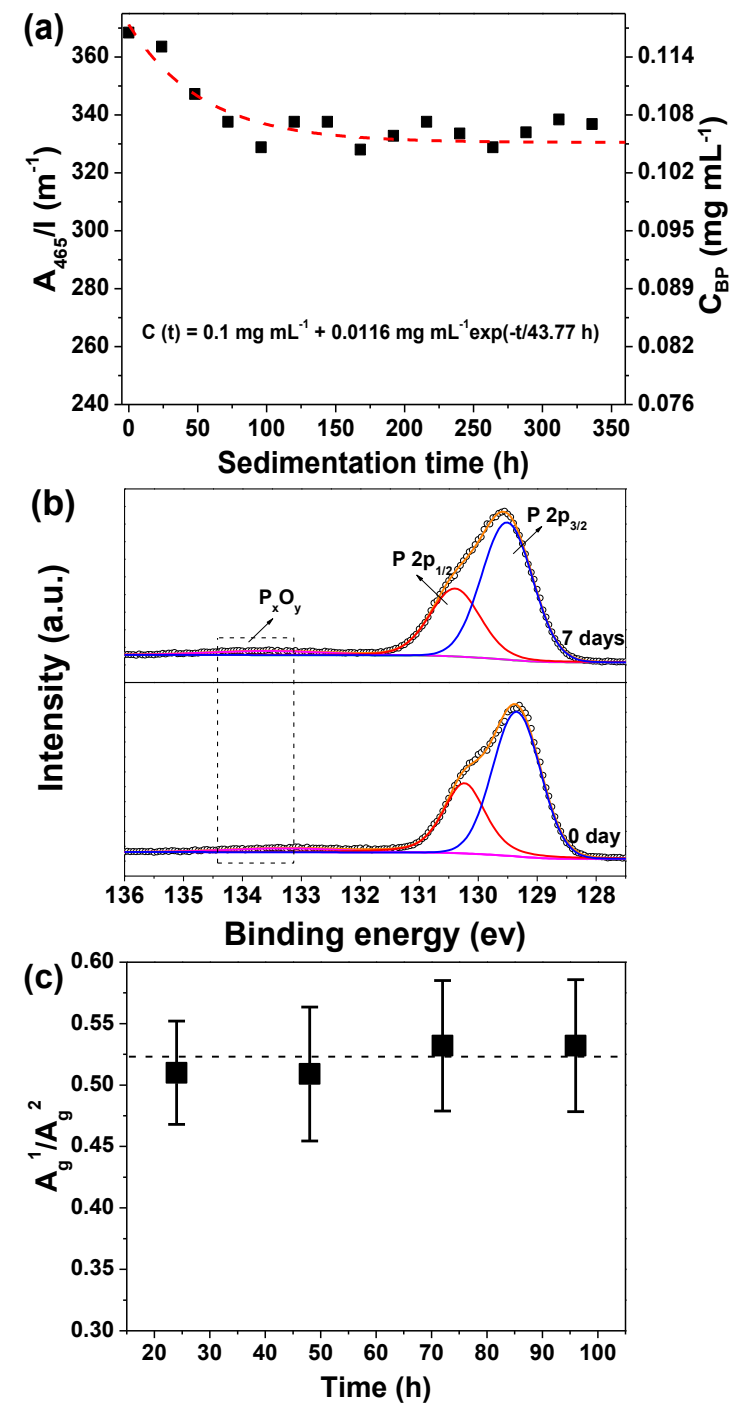

Figure 6. (a) $C_{\mathrm{BP}}$ versus sedimentation time for a phosphorene dispersion in a glovebox $\left(C_{\mathrm{I}}=1 \mathrm{mg} \mathrm{mL}{ }^{-1} ; t_{\text {sonic }}=12 \mathrm{~h} ; \mathrm{CF}: 3000 \mathrm{rpm}, 30 \mathrm{~min}\right)$. The dashed line is the fitting of the data. (b) P 2p core-level XPS spectra of BP nanosheets at 0 day, and 7 days ambient 
exposure. (c) Time evolution of Raman intensity ratio of the $\mathrm{Ag}_{\mathrm{g}}{ }^{1} / \mathrm{Ag}^{2}$ modes for BP nanosheet powder after exposure to air, obtained from the analysis of 441 baselinecorrected spectra acquired over an area of $25 \times 25 \mu \mathrm{m}^{2}$ in each case.

Raman spectra of BP nanosheets were monitored at 24, 48, 72, and $96 \mathrm{~h}$ after exposure to air at $\lambda=532 \mathrm{~nm}$. Raman maps and the statistic revealed the average values of $\mathrm{Ag}_{\mathrm{g}}{ }^{1} / \mathrm{Ag}^{2}>0.5$ in all cases characteristic of low oxidation levels (Fig. S8). The Raman intensity ratio of $\mathrm{Ag}_{\mathrm{g}}{ }^{1} \mathrm{Ag}^{2}$ remained nearly unchanged after $96 \mathrm{~h}$ exposure (Fig. 6c), suggesting no occurrence of degradation, in accordance with XPS results.
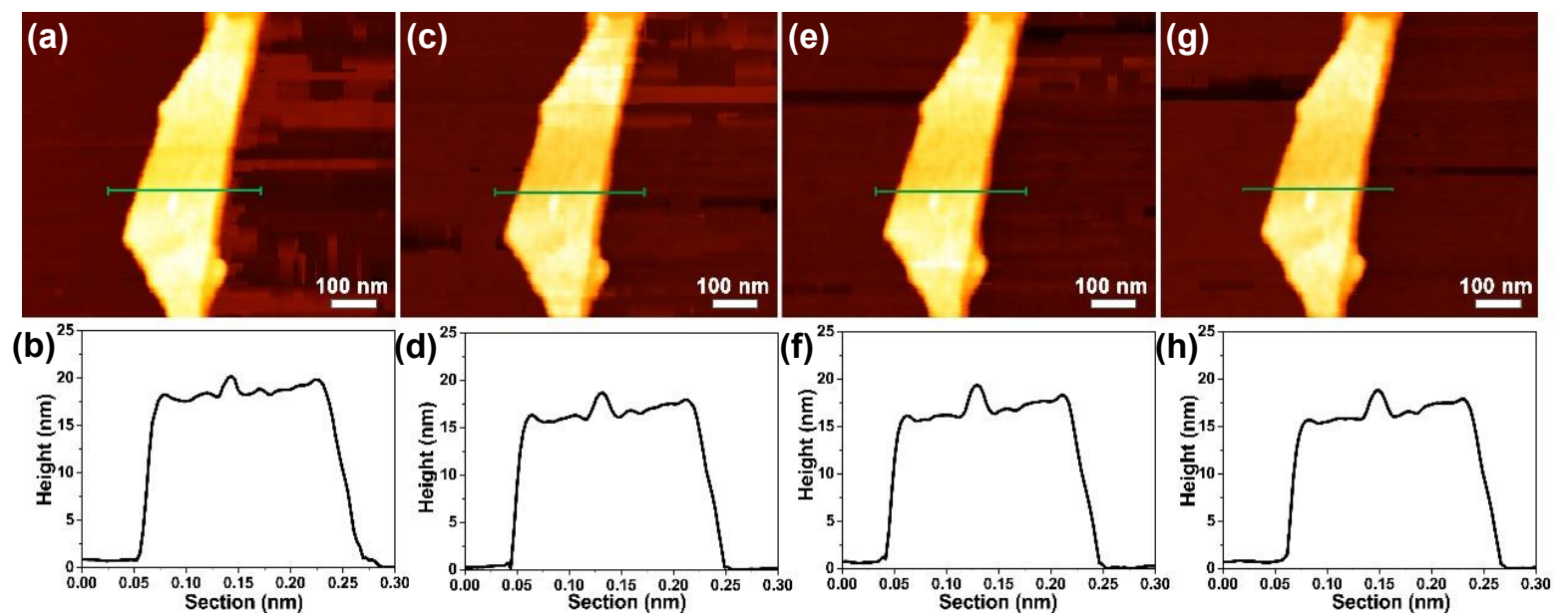

Figure 7. AFM images along with AFM height profiles of an exfoliated BP flake $\left(C_{\mathrm{I}}=\right.$ $1 \mathrm{mg} \mathrm{mL}{ }^{-1} ; t_{\text {sonic }}=12 \mathrm{~h}$; CF: $3000 \mathrm{rpm}, 30 \mathrm{~min}$ ) on a $\mathrm{SiO}_{2} / \mathrm{Si}$ substrate after exposure to ambient conditions with an average temperature of $25^{\circ} \mathrm{C}$ and a relative humidity of $100 \%$ for $(\mathrm{a}, \mathrm{b}) 0 \mathrm{~h},(\mathrm{c}, \mathrm{d}) 4 \mathrm{~h},(\mathrm{e}, \mathrm{f}) 8 \mathrm{~h}$, and $(\mathrm{g}, \mathrm{h}) 12 \mathrm{~h}$. Those brighter dots on the surface of the flake shown in a, c, e, and g may originate from the solvent DMPU. No bubble formation in the flake indicative of irreversible BP oxidation was observed after $12 \mathrm{~h}$ in ambient conditions.

To gain insight into the ambient degradation behavior of exfoliated BP nanosheets in DMPU, we performed AFM measurements. Fig. 7 shows AFM images along with AFM height profiles of a16 nm-thick BP flake after exposure to air for 0, 4, 8, and 12 h. No signs of apparent degradation are observable even after $12 \mathrm{~h}$ of ambient exposure with an average temperature of $25^{\circ} \mathrm{C}$ and a relative humidity of $100 \%$. 

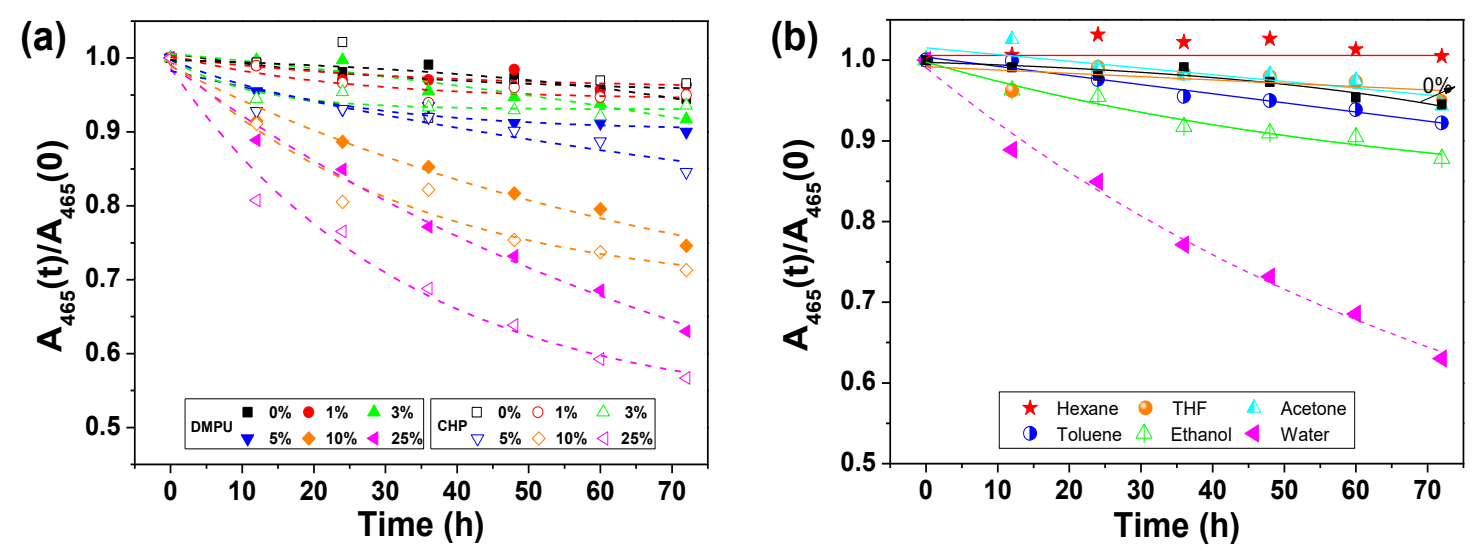

Figure 8. (a) Relative absorbance, $A_{465}(t) / A_{465}(0)$, monitoring as a function of time $(t)$ for phosphorene dispersions with varying volume fractions of water added. The relative absorbances for BP dispersions in CHP with addition of $0,1,3,5,10$, and $25 \% \mathrm{H}_{2} \mathrm{O}$ were added for comparison. (b) $A_{465}(t) / A_{465}(0)$ versus time ( $t$ ) for phosphorene dispersions with addition of $25 \%$ volume fractions of hexane, THF, acetone, toluene, ethanol, and water.

We further tested the stability of phosphorene dispersions by purposeful addition of varying proportions of water through monitoring the variation of absorbance $(A)$ versus time (Fig. 8a and Fig. S9). Only a slight fall (less than 5\%) in measured absorbance occurred for the dispersion in DMPU without addition of water, similar to the dispersion in CHP. Although the absorbance decreased with increasing the volume fraction of water, the dispersions in DMPU demonstrated slower degradation kinetics with equivalent addition of water compared with the dispersions in CHP. Such degradation may result from an acid-base disproportionation reaction predominantly involving nanosheet edges $\left[\mathrm{BP}+3 \mathrm{H}_{2} \mathrm{O} \rightarrow \mathrm{BP}_{2 \mathrm{VAC}}\right.$ (a defective structure with two $\mathrm{P}$ vacancies formed in the $\mathrm{BP}$ supercell) $\left.+\mathrm{PH}_{3}+\mathrm{H}_{3} \mathrm{PO}_{3}\right],{ }^{[42]}$ which can be accelerated in the presence of air. We fitted these curves by monoexponential decays: $A=A_{\text {Unre }}+A_{\mathrm{Re}}$ $\mathrm{e}^{-t / \tau}$, where $A_{\text {Unre }}$ represents the amounts of BP that reacted over time, and $A_{\text {Re }}$ represents those BP that did not react, while $t$ and $\tau$ represent time and decay time. The values of $\tau$ derived are in the range of 50-128 $\mathrm{h}$, which are significantly higher than the decay 
time of $1 \mathrm{~h}$ observed for mechanically cleaved BP in water. ${ }^{[42]}$ This implies that the DMPU solvation shell can act as a barrier to prevent oxidative species reaching the nanosheet surface and dissociation of oxygen molecules, with higher protection efficiency than the best stable solvent reported hitherto.

We also made a preliminary investigation into some other common solvents regarding their effects on the dispersion of nanosheets in DMPU (Fig. 8b). Interestingly, the BP dispersion remained substantially stable over $60 \mathrm{~h}$ even when $25 \%$ volume fraction of hexane was added. Addition of tetrahydrofuran (THF), acetone, toluence, and ethanol may induce aggregation, but no sedimentation was observed after $60 \mathrm{~h}$. Note that the degrees of dispersion degradation are less than $10 \%$ in all cases, significantly lower than that in water. We believe that these results will aid the practical handling of BP dispersions.

(a)

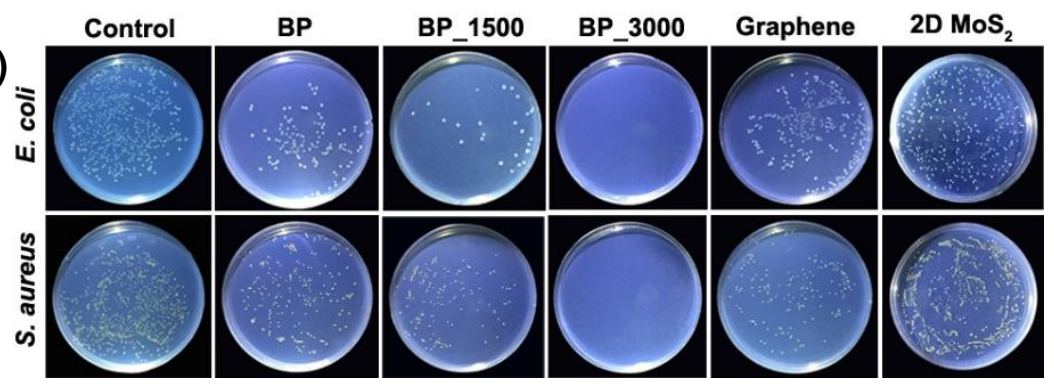

(c)

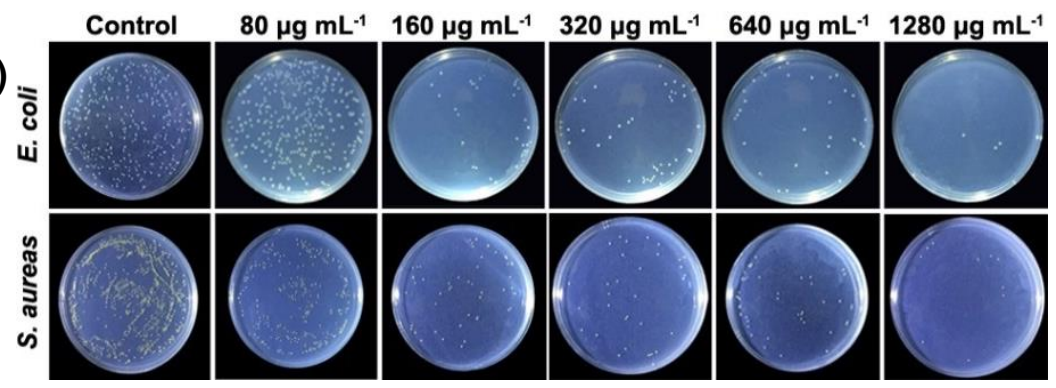

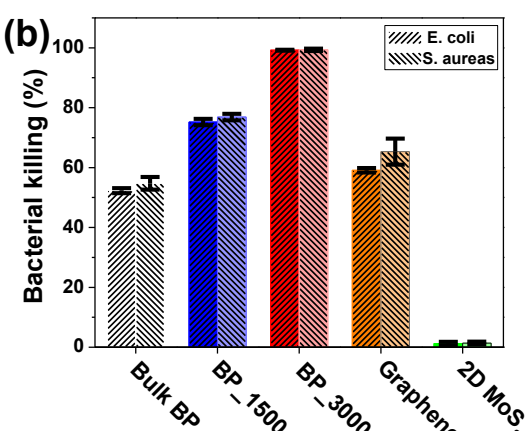

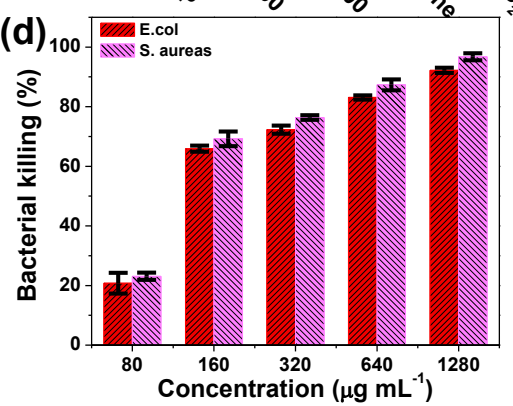

Figure 9. (a) Photographs of bacterial colonies of E. coli (top panel) and S. aureas (bottom panel) on agar plates after treatment with bulk BP, BP_1500 and BP_3000 nanosheets, graphene, and $2 \mathrm{D} \mathrm{MoS}_{2}$. (b) Bacterial killing ratios of bulk BP, BP_1500 and $\mathrm{BP} 3000$ nanosheets, graphene, and $2 \mathrm{D} \mathrm{MoS}_{2}$ under the same experimental conditions. (c) Photographs of bacterial colonies of E. coli (top panel) and S. aureas (bottom panel) on agar plates after treatment with varying concentrations of BP_3000. 
(d) Bacterial killing ratio as a function of $\mathrm{BP} \_3000$ concentration. The minimum inhibitory concentration (MIC) was found to be $\sim 160 \mu \mathrm{g} \mathrm{mL}^{-1}$. The irradiation time in all cases was 3 min.

Antibacterial application. Compared with other 2D materials such as graphene and transition metal dichalcogenides (TMDs), BP has higher surface to volume ratio owing to its puckered lattice configuration. Of particular interest is that BP possesses unique optical absorptions which can be broadly tuned by tailoring the number of stacked layers. These aspects provide opportunities for using BP nanosheets as a photothermal agent in cancer therapy. ${ }^{[26-28,63-65]}$ Despite this being the case, the antimicrobial activities of BP and exfoliated BP have been rarely reported. Here, we report a study on the antibacterial properties of BP using a LB-agar coated plate counting method.

Gram-negative Escherichia coli (E. coli) and Gram-positive Staphylococcus aureus ( $S$. aureas) that cause serious infections were chosen as microbial models for antibacterial activity evaluation. We compared the antibacterial activities of our exfoliated BP nanosheets with bulk BP, graphene, and 2D MoS 2 (IPA exfoliated). As depicted in Fig. 9a and b, 2D $\mathrm{MoS}_{2}$ showed negligible bacterial killing ability under

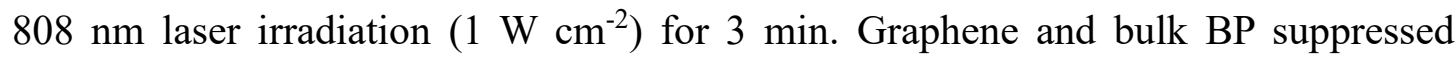
bacterial growth but with bacterial killing ratios of less than $60 \%$. In stark contrast, BP nanosheets exhibited remarkable antibacterial capabilities with killing ratios against both $E$. coli and $S$. aureas as high as about $99.2 \%$. Interestingly, the BP nanosheets attained at a higher CF speed killed more bacteria and showed the best antibacterial activity among all employed materials. Such an outstanding performance is most likely due to its better optical absorption in the near-infrared region originating from the thinner thickness of BP obtained at higher CF speeds. Furthermore, we investigated the effects of irradiation time and BP concentration on photothermal efficiency. When irradiated with $808 \mathrm{~nm}$ laser for $10 \mathrm{~min}$, bulk BP, exfoliated BP nanosheets, and graphene all killed gram-negative and gram-positive bacteria, whereas $2 \mathrm{D} \mathrm{MoS}_{2}$ did not show effective antibacterial effect, as can be clearly seen from Fig. S10. When the irradiation time was reduced to $2 \mathrm{~min}$, no antibacterial activities were observed for 
graphene and bulk BP, but BP nanosheets killed over half bacteria showing effective bacterial inhibition. The concentration dependent antibacterial activities of BP were also studied. Fig. 9d shows that the bacterial cell viability loss (killing ratio) exposed to $\mathrm{BP}$ nanosheet concentrations in the range of $80-1280 \mu \mathrm{g} \mathrm{mL}^{-1}$. The bacterial cell loss gradually increased with the increase of BP concentration. More than $65 \%$ bacterial viability loss for both E. coli and $S$. aureas was observed at $160 \mu \mathrm{g} \mathrm{mL} \mathrm{m}^{-1}$ of BP nanosheets obtained at $3000 \mathrm{rpm}$ (BP_3000) within 3 min of irradiation (Fig. 9c and d). Additionally, BP nanosheets have a slightly stronger influence on $S$. aureas than E. coli. 


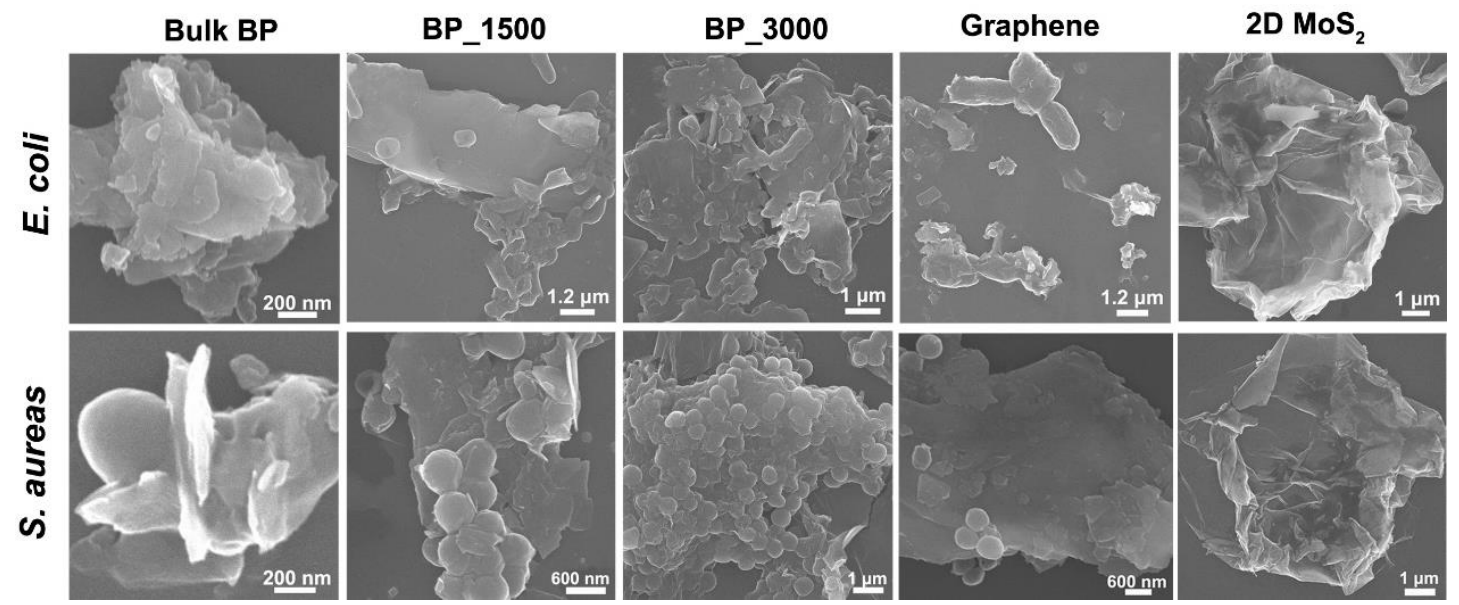

Figure 10. SEM images of E. coli (top panel) and S. aureas (bottom panel) treated with bulk BP, BP_1500 and BP_3000 nanosheets, graphene, and 2D $\mathrm{MoS}_{2}$, respectively. Control bacterial cells were viable with no observed membrane damage or cell death.

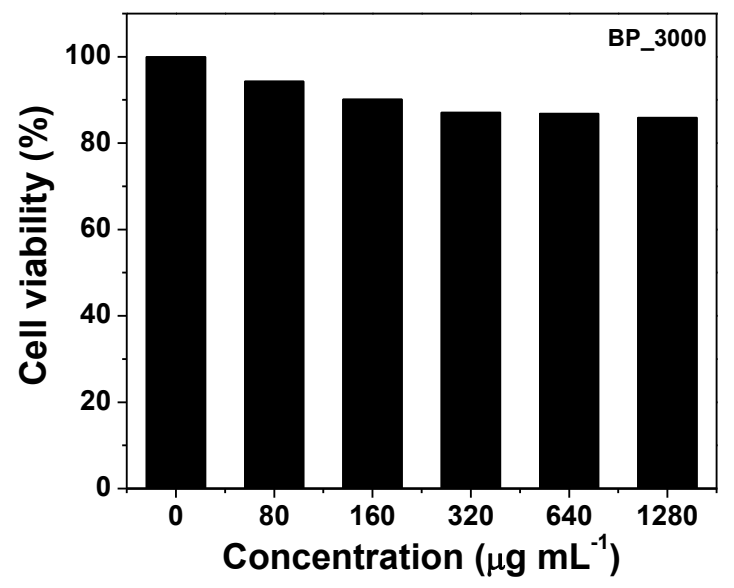

Figure 11. Cell viability of HeLa cells treated with different concentrations of BP_3000 nanosheets for $24 \mathrm{~h}$.

To understand the antibacterial effects of bulk and BP nanosheets, graphene, and 2D $\mathrm{MoS}_{2}$, changes of morphology and membrance integrity of E. coli and $S$. aureas cells after laser exposure were examine by SEM. As revealed by SEM images in Fig. 10, bacterial cells of both $E$. coli and $S$. aureas cultured in the presence of BP nanosheets shrinked. BP nanosheets with more accessible surface area than bulk BP favor trapping or wrapping of microbes and subsequent formation of agglomerates. More bacteria were observed to aggregate on or around BP nanosheets relative to bulk BP, graphene, and 2D $\mathrm{MoS}_{2}$, leading to enhanced antibacterial efficiency. Additionally, the 
hydrophilic BP nanosheets could effectively attach to bacteria, facilitating their photothermal inactivation. Moreover, BP_3000 appears to adsorb more bacteria than the one obtained at $1500 \mathrm{rpm}\left(\mathrm{BP}_{-} 1500\right)$, consistent with the LB-agar plate results. The biocompatibility of BP nanosheets, a crucial factor in biomedical area, was evaluated by a MTT method with HeLa cells. The cytotoxicity of BP nanosheets was calculated, as shown in Fig. 11. Although the cell viability decreased with increasing the dosage of $\mathrm{BP}$ nanosheets, it still remained a rather high level $(\sim 86 \%)$ even at a high BP concentration $\left(1280 \mu \mathrm{g} \mathrm{mL}^{-1}\right)$, indicating small cytotoxicity of the BP nanosheets.

\section{Conclusion}

We have shown a new and harmless molecule, $N, N^{\prime}$-dimethylpropyleneurea (DMPU), that enables efficient exfoliation and dispersion of BP, outperforming all other reported solvents. Exfoliated nanosheets are highly crystalline and free of defects. The BP nanosheets are remarkably stable with an oxide content of only 4.3 atom \% even after exposure to air for 7 days, probably due to protection by the solvent solvation shell. Such DMPU-stabilized BP nanosheets showed higher resistance against degradation with addition of water or other common solvents than the best solvent system reported so far, cyclohexyl-2-pyrrolidinone (CHP) exfoliated BP. Furthermore, we demonstrate that exfoliated BP nanosheets exhibited thickness-dependent photothermal antibacterial properties with higher efficiency than graphene and two-dimensional $\mathrm{MoS}_{2}$ while showing minimal cytotoxicity. Our results suggest that the solvent-stabilized BP nanosheets with good biocompatibility have large potential as bacteriostatic agents.

\section{Acknowledgments}

This work was supported by the State Key Laboratory of Organic-Inorganic Composites (No. oic-201503005); the Fundamental Research Funds for the Central Universities (No. buctrc201525); Beijing National Laboratory for Molecular Sciences (BNLMS20160133); Key Laboratory of Photochemical Conversion and Optoelectronic Materials, TIPC, CAS. Z. Wang acknowledges the support from Natural Science Foundation of China (grant No. 21575032, 21775010, 81728010); the Fundamental 
Research Funds for the Central Universities (PYBZ1707, buctrc201607); Beijing Natural Science Foundation - Haidian Original Innovation Joint Fund (L172018). 


\section{Reference}

[1] H. Liu, A. T. Neal, Z. Zhu, Z. Luo, X. F. Xu, D. Tomanek, P. D. Ye, ACS Nano 2014, 8, 4033 .

[2] R. Gusmao, Z. Sofer, M. Pumera, Angew. Chem. Int. Ed. 2017, 56, 8052.

[3] Y. Q. Zhang, N. N. Dong, H. C. Tao, C. Yan, J. W. Huang, T. F. Liu, A. W. Robertson, J. Texter, J. Wang, Z. Y. Sun, Chem. Mater. 2017, 29, 6445.

[4] M. Bat-Erdene, M. Batmunkh, C. J. Shearer, S. A. Tawfik, M. J. Ford, L. Yu, A. J. Sibley, A. D. Slattery, J. S. Quinton, C. T. Gibson, J. G. Shapter, Small Methods 2017, 1700260.

[5] A. H. Castro Neto, F. Guinea, N. M. R. Peres, K. S. Novoselov, A. K. Geim, Rev. Mod. Phys. 2009, 81, 109.

[6] Q. H. Wang, K. Kalantar-Zadeh, A. Kis, J. N. Coleman, M. S. Strano, Nat. Nanotechnol. 2012, 7, 699.

[7] V. Tran, R. Soklaski, Y. F. Liang, L. Yang, Phys. Rev. B 2014, 89, 817.

[8] L. K. Li, Y. J. Yu, G. J. Ye, Q. Q. Ge, X. D. Ou, H. Wu, D. L. Feng, X. H. Chen, Y. B. Zhang, Nat. Nanotechnol. 2014, 9, 372.

[9] J. S. Qiao, X. H. Kong, Z. X. Hu, F. Yang, W. Ji, Nat. Commun. 2014, 5, 4475.

[10] K. P. Wang, B. M. Szydlowska, G. Z. Wang, X. Y. Zhang, J. J. Wang, J. J. Magan, L. Zhang, J. N. Coleman, J. Wang, W. J. Blau, ACS Nano 2016, 10, 6923.

[11]S. P. Koenig, R. A. Doganov, H. Schmidt, A. H. C. Neto, B. Ozyilmaz, Appl. Phys. Lett. 2014, 104, 10451.

[12] A. N. Abbas, B. L. Liu, L. Chen, Y. Q. Ma, S. Cong, N. Aroonyadet, M. Kopf, T. Nilges, C. W. Zhou, ACS Nano 2015, 9, 5618.

[13] G. Lee, S. Kim, S. Jung, S. Jang, J. Kim, Sens. Actuators B Chem. 2017, 250, 569.

[14] P. Yasaei, A. Behranginia, T. Foroozan, M. Asadi, K. Kim, F. Khalili-Araghi, A. Salehi-Khojin, ACS Nano 2015, 9, 9898.

[15] S. Y. Cho, H. J. Koh, H. W. Yoo, H. T. Jung, Chem. Mater. 2017, 29, 7197.

[16] M. Zhu, X. Cai, M. Fujitsuka, J. Zhang, T. Majima, Angew. Chem. Int. Ed. 2017, 56, 2064.

[17]X. Zhu, T. Zhang, Z. Sun, H. Chen, G. Jian, C. Xiang, H. Ji, P. Du, S. Yang, Adv. Mater. 2017, 29, 1605776.

[18] M. Zhu, Y. Osakada, S. Kim, M. Fujitsuka, T. Majima, App1. Catal. B Envir. 2017, $217,285$.

[19] Q. Q. Jiang, L. Xu, N. Chen, H. Zhang, L. M. Dai, S. Y. Wang, Angew. Chem. Int. Ed. 2016, 55, 13849.

[20]X. Ren, J. Zhou, X. Qi, Y. Liu, Z. Huang, Z. Li, Y. Ge, S. C. Dhanabalan, J. S. Ponraj, S. Wang, Adv. Energy Mater. 2017, 1700396.

[21] M. Zhu, S. Kim, L. Mao, M. Fujitsuka, J. Zhang, X. Wang, T. Majima, J. Am. Chem. Soc. 2017, 139, 13234.

[22] J. Hu, Z. K. Guo, P. E. McWilliams, J. E. Darges, D. L. Druffel, A. M. Moran, S. C. Warren, Nano Lett. 2016, 16, 74.

[23] L. Chen, G. M. Zhou, Z. B. Liu, X. M. Ma, J. Chen, Z. Y. Zhang, X. L. Ma, F. Li, H. M. Cheng, W. C. Ren, Adv. Mater. 2016, 28, 510. 
[24] X. Yue, S. Yi, R. Wang, Z. Zhang, S. Qiu, Small 2017, 13, 1603301.

[25] T. Chen, P. Zhao, X. Guo, S. Zhang, Nano Lett. 2017, 17, 2299.

[26]Z. Sun, H. Xie, S. Tang, X. F. Yu, Z. Guo, J. Shao, H. Zhang, H. Huang, H. Wang, P. K. Chu, Angew. Chem. Int. Ed. 2015, 54, 11526.

[27]W. Tao, X. B. Zhu, X. H. Yu, X. W. Zeng, Q. L. Xiao, X. D. Zhang, X. Y. Ji, X. S. Wang, J. J. Shi, H. Zhang, L. Mei, Adv. Mater. 2017, 29, 1603276.

[28] J. Shao, H. Xie, H. Huang, Z. Li, Z. Sun, Y. Xu, Q. Xiao, X. F. Yu, Y. Zhao, H. Zhang, Nat. Commun. 2016, 7, 12967.

[29] W. Chen, J. Ouyang, H. Liu, M. Chen, K. Zeng, J. Sheng, Z. Liu, Y. Han, L. Wang, J. Li, Adv. Mater. 2016, 29, 1603864.

[30] H. C. Tao, Y. N. Gao, N. Talreja, F. Guo, J. Texter, Z. Y. Sun, C. Yan, J. Mater. Chem. A 2017, 5, 7257.

[31] G. Sansone, L. Maschio, D. Usvyat, M. Schutz, A. Karttunen, J. Phys. Chem. Lett. 2016, 7, 131.

[32] V. Sresht, A. A. Padua; D. Blankschtein, ACS Nano 2015, 9, 8255.

[33] V. Nicolosi, M. Chhowalla, M. G. Kanatzidis, M. S. Strano, J. N. Coleman, Science 2013, 340, 1226419.

[34] A. Ciesielski, P. Samori, Chem. Soc. Rev. 2014, 43, 381.

[35] H. C. Tao, Y. Q. Zhang, Y. N. Gao, Z. Y. Sun, C. Yan, J. Texter, Phys. Chem. Chem. Phys. 2017, 19, 921.

[36] C. Backes, T. M. Higgins, A. Kelly, C. Boland, A. Harvey, D. Hanlon, J. N. Coleman, Chem. Mater. 2017, 29, 243.

[37] Z. Y. Sun, J. Vivekananthan, D. A. Guschin, X. Huang, V. Kuznetsov, P. Ebbinghaus, A. Sarfraz, M. Muhler, W. Schuhmann, Chem. Eur. J. 2014, 20, 5752.

[38] J. R. Brent, N. Savjani, E. A. Lewis, S. J. Haigh, D. J. Lewis, P. 0’Brien, Chem. Commun. 2014, 50, 13338.

[39] S. H. Lin, S. H. Liu, Z. B. Yang, Y. Y. Li, T. W. Ng, Z. Q. Xu, Q. L. Bao, J. H. Hao, C. S. Lee, C. Surya, F. Yan, S. P. Lau, Adv. Funct. Mater. 2016, 26, 864.

[40] J. Kang, J. D. Wood, S. A. Wells, J. H. Lee, X. L. Liu, K. S. Chen, M. C. Hersam, ACS Nano 2015, 9, 3596.

[41] D. J. Late, Micropor. Mesopor. Mater. 2016, 225, 494.

[42] D. Hanlon, C. Backes, E. Doherty, C. S. Cucinotta, N. C. Berner, C. Boland, K. Lee, A. Harvey, P. Lynch, Z. Gholamvand, S. Zhang, K. Wang, G. Moynihan, A. Pokle, Q. M. Ramasse, N. McEvoy, W. J. Blau, J. Wang, G. Abellan, F. Hauke, A. Hirsch, S. Sanvito, D. D. O’Regan, G. S. Duesberg, V. Nicolosi, J. N. Coleman, Nat. Commun. $2015,6,8563$.

[43] A. H. Woomer, T. W. Farnsworth, J. Hu, R. A. Wells, C. L. Donley, S. C. Warren, ACS Nano 2015, 9, 8869.

[44] P. Yasaei, B. Kumar, T. Foroozan, C. Wang, M. Asadi, D. Tusche1, J. E. Indacochea, R. F. Klie, A. Salehi-Khojin, Adv. Mater. 2015, 27, 1887.

[45] C. X. Hao, F. S. Wen, J. Y. Xiang, S. J. Yuan, B. C. Yang, L. Li, W. H. Wang, Z. M. Zeng, L. M. Wang, Z. Y. Liu, Y. J. Tian, Adv. Funct. Mater. 2016, 26, 2016.

[46] C. Hao, B. Yang, F. Wen, J. Xiang, L. Li, W. Wang, Z. Zeng, B. Xu, Z. Zhao, Z. Liu, Adv. Mater. 2016, 28, 3194. 
[47] J. Kang, S. A. Wells, J. D. Wood, J. H. Lee, X. Liu, C. R. Ryder, J. Zhu, J. R. Guest, C. A. Husko, M. C. Hersam, PNAS 2016, 113, 11688.

[48] Y. Zhang, N. Dong, H. Tao, C. Yan, J. Huang, T. Liu, A. W. Robertson, J. Texter, J. Wang, Z. Sun, Chem. Mater. 2017, 29, 6445.

[49] W. C. Zhao, Z. M. Xue, J. F. Wang, J. Y. Jiang, X. H. Zhao, T. C. Mu, ACS Appl. Mater. Interfaces 2015, 7, 27608.

[50] V. V. Chaban, E. E. Fileti, 0. V. Prezhdo, ACS Nano 2017, 11, 6459.

[51] R. R. Nair, P. Blake, A. N. Grigorenko, K. S. Novoselov, T. J. Booth, T. Stauber, N. M. R. Peres, A. K. Geim, Science 2008, 320, 1308.

[52] J. Texter, Angew. Chem. Int. Ed. 2015, 54, 10258.

[53] A. Castellanos-Gomez, L. Vicarelli, E. Prada, J. 0. Island, K. L. NarasimhaAcharya, S. I. Blanter, D. J. Groenendijk, M. Buscema, G. A. Steele, J. V. Alvarez, H. W. Zandbergen, J. J. Palacios, H. S. J. van der Zant, 2D Mater. 2014, 1, 025001. [54] P. Nemes-Incze, Z. Osvath, K. Kamaras, L. P. Biro, Carbon 2008, 46, 1435.

[55] A. Favron, E. Gaufres, F. Fossard, A. L. Phaneuf-L' Heureux, N. Y. Tang, P. L. Levesque, A. Loiseau, R. Leonelli, S. Francoeur, R. Martel, Nat. Mater. 2015, 14, 826.

[56] Z. Guo, H. Zhang, S. Lu, Z. Wang, S. Tang, J. Shao, Z. Sun, H. Xie, H. Wang, X. F. Yu, Adv. Funct. Mater. 2015, 25, 6996.

[57]W. L. Jorgensen, D. S. M. And, J. Tiradorives, J. Am. Chem. Soc. 1996, 118, 11225.

[58] S. Plimpton, J. Comput. Phys. 1995, 117, 1.

[59] Y. Huang, J. S. Qiao, K. He, S. Bliznakov, E. Sutter, X. J. Chen, D. Luo, F. K. Meng, D. Su, J. Decker, W. Ji, R. S. Ruoff, P. Sutter, Chem. Mater. 2016, 28, 8330. [60]G. Abellán, S. Wild, V. Lloret, N. Scheuschner, R. Gillen, U. Mundloch, J. Maultzsch, M. Varela, F. Hauke, A. Hirsch, J. Am. Chem. Soc. 2017, 139, 10432.

[61] C. R. Ryder, J. D. Wood, S. A. Wells, M. C. Hersam, ACS Nano 2016, 10, 3900.

[62] H. Kwon, S. W. Seo, T. G. Kim, E. S. Lee, P. T. Lanh, S. Yang, S. Ryu, J. W. Kim, ACS Nano 2016, 10, 8723.

[63] W. S. Chen, J. Ouyang, H. Liu, M. Chen, K. Zeng, J. P. Sheng, Z. J. Liu, Y. J. Han, L. Q. Wang, J. Li, L. Deng, Y. N. Liu, S. J. Guo, Adv. Mater. 2017, 29, 1603864. [64] Y. T. Zhao, L. P. Tong, Z. B. Li, N. Yang, H. D. Fu, L. Wu, H. D. Cui, W. H. Zhou, J. H. Wang, H. Y. Wang, P. K. Chu, X. F. Yu, Chem. Mater. 2017, 29, 7131.

[65] J. D. Shao, H. H. Xie, H. Huang, Z. B. Li, Z. B. Sun, Y. H. Xu, Q. L. Xiao, X. F. Yu, Y. T. Zhao, H. Zhang, H. Y. Wang, P. K. Chu, Nat. Commun. 2016, 7, 12967. 